\title{
Heat Removal for Adsorbed Natural Gas Storage - Lab to Industrial Scale
}

Yuguo Wang, George P. Lithoxoos, Rashid M. Othman

Research \& Development Center, Saudi Arabia Oil Company, Dhahran, 31311, Saudi Arabia

Number of pages: 9

Number of figures: 4

Number of tables: 5 
Table S1. Physical properties of the adsorbent used in the heat removal optimization study.

\begin{tabular}{|c|c|c|c|c|c|}
\hline Sample & $\mathrm{AC}_{1}$ & $\mathrm{AC} 2$ & $\mathrm{AC}_{3}$ & $\mathrm{AC}_{4}$ & $\mathrm{AC}_{5}$ \\
\hline ASTM mesh size & $8 \times 16$ & $30 \times 70$ & $2 \times 60$ & $12 \times 40$ & $6 \times 60$ \\
\hline Bulk density $\left(\mathrm{g} / \mathrm{cm}^{3}\right)$ & 0.47 & 0.39 & 0.49 & 0.54 & 0.50 \\
\hline Skeletal density $\left(\mathrm{g} / \mathrm{cm}^{3}\right)$ & 2.299 & 2.363 & 2.402 & 2.059 & 2.286 \\
\hline \multicolumn{6}{|c|}{ Nitrogen Porosimetry (77 K) } \\
\hline t-plot micropore volume $\left(\mathrm{cm}^{3} / \mathrm{g}\right)$ & 0.417 & 0.412 & 0.487 & 0.292 & $0.45^{2}$ \\
\hline t-plot micropore area $\left(\mathrm{m}^{2} / \mathrm{g}\right)$ & 968.6 & 969.4 & 1186.1 & 722.5 & 1082.1 \\
\hline BET surface area $\left(\mathrm{m}^{2} / \mathrm{g}\right)$ & 1235 & 1589 & 1426 & 999 & 1510 \\
\hline Total Pore Volume $\left(\mathrm{cm}^{3} / \mathrm{g}\right)$ & 0.629 & 0.747 & 0.599 & 0.500 & 0.682 \\
\hline BJH average pore width $(\AA)$ & 18.00 & 18.70 & $17 \cdot 47$ & 20.64 & 26.08 \\
\hline \multicolumn{6}{|c|}{ Results of Dubinin-Astakhov Modeling of Methane Adsorption Data at 10, 21, 38 and $56^{\circ} \mathrm{C}$. } \\
\hline & $\mathrm{AC}_{1}$ & $\mathrm{AC}_{2}$ & $\mathrm{AC}_{3}$ & $\mathrm{AC}_{4}$ & $\mathrm{AC}_{5}$ \\
\hline Micropore volume $\left(\mathrm{cm}^{3} / \mathrm{g}\right)$ & 0.380 & 0.449 & 0.416 & 0.2865 & 0.445 \\
\hline Micropore average pore width $(\AA)$ & 9.44 & 10.62 & 9.88 & 10.34 & $9 \cdot 58$ \\
\hline
\end{tabular}

In Table $\mathrm{S}$, the total pore volume from nitrogen porosimetry refers to the pores that have diameter range of o to $20 \mathrm{~nm}$ and the micropores are defined as those having a diameter range of o to $2 \mathrm{~nm}$. For the mercury porosimetry, mesopore volume refers to the pores with a width range of 2 to $50 \mathrm{~nm}$ and to the macropores with a width range of 50 to $10000 \mathrm{~nm}$.

Characterization. Nitrogen adsorption at ${ }_{77} \mathrm{~K}$ data is presented in Table S2. Nitrogen adsorption at ${ }_{77} \mathrm{~K}$ was used to determine the surface area and pore volume of activated carbon samples. Figure S1 shows the plot of the amount of nitrogen adsorbed versus the relative pressure. The shape of these five isotherms are of Type-I which indicates that these activated carbons are essentially microporous. After the relative pressure of 0.8 , there is a slight increase in nitrogen adsorption. This is most probably due to the presence of mesopores, where the condensation of nitrogen occurs as the pressure goes up.

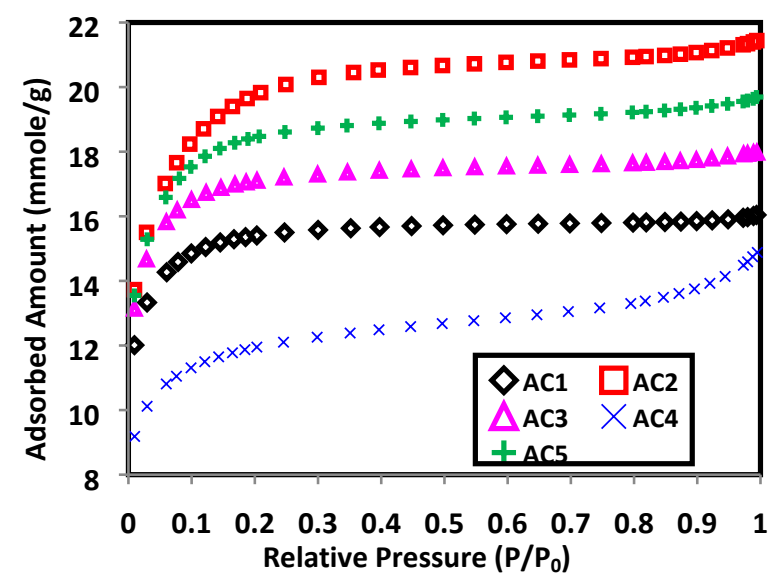

Figure $\mathrm{S}_{1} . \mathrm{N}_{2}$ adsorption isotherms at $77 \mathrm{~K}$ on granular activated carbons (data in Table $\mathrm{S}_{3}$ ). 
Experimental Adsorption Isotherms. Figures S2.1 and S2.2 show the plots of the amount of adsorbed methane versus pressure at $21^{\circ} \mathrm{C}$ for the five different activated carbons (data in Table $\mathrm{S}_{3}$ ). All the plots have the shape of Type-I isotherm, which also indicates that these samples are microporous materials, typical of activated carbons. In Figure 4.2 the volumetric data are based on volume to volume ratio (V/V).

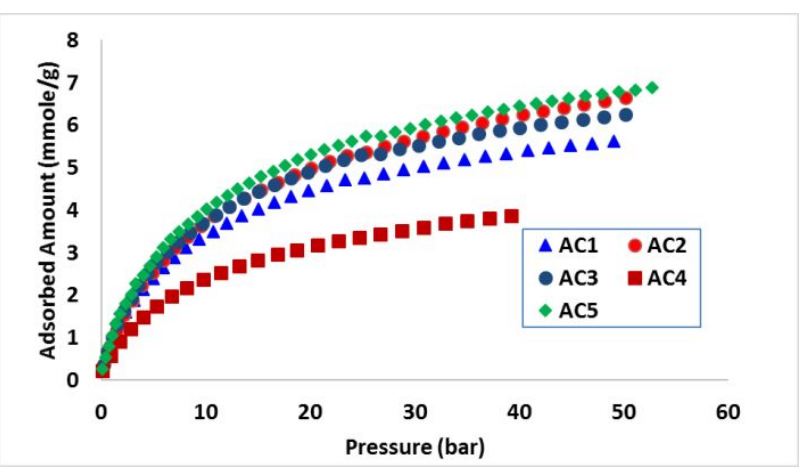

Figure S2.1. Comparison of mass to mass ( $\mathrm{mmol} / \mathrm{g}$ ) adsorption capacity among the five granular activated carbons.

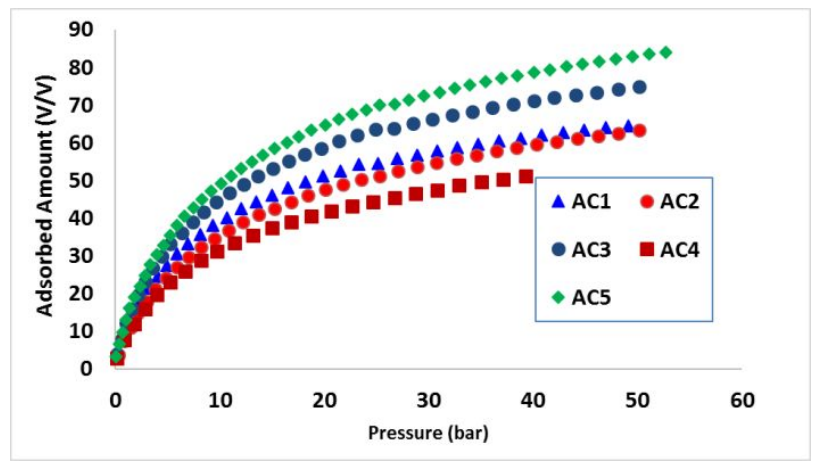

Figure S2.2. Comparison of volume to volume (v/v) adsorption capacity among the five granular activated carbons.

Based on the moles of methane adsorbed per gram of activated carbon in Figure S2.1, the capacity of methane adsorption on five activated carbons increases in the order of $\mathrm{AC}_{4}<\mathrm{AC}_{1}<\mathrm{AC}_{3}<\mathrm{AC}_{2}<\mathrm{AC}$. Although the surface areas of $A C_{2}$ and $A C_{5}$ are close to each other, the micropore volume of $A_{5}\left(0.452 \mathrm{~cm}^{3} / \mathrm{g}\right)$ is higher than that of $\mathrm{AC}_{2}\left(0.412 \mathrm{~cm}^{3} / \mathrm{g}\right)$, which is important for the adsorption on microporous adsorbents occurring by the mechanism of pore filling. This may also be due to the fact that $\mathrm{AC}_{5}$ has more slit pores that are close to the optimal pore size for methane adsorption. Earlier theoretical studies ${ }^{4-43}$ found that for methane adsorption on slit-pore activated carbon, the optimum pore width is 11.2 to $11.4 \AA$ in order to create the maximum density for the adsorbed phase.

Based on the volume of methane adsorbed per volume of activated carbon presented in Figure $\mathrm{S}_{2.2}$, the capacity for methane adsorption increases in the order of $\mathrm{AC}_{4}<\mathrm{AC}_{2}<\mathrm{AC}_{1}<\mathrm{AC}_{3}<\mathrm{AC}_{5}$. This change of order in comparison to the mass to mass adsorption capacity in Figure S2.1 can be attributed to the fact that the bulk density in volume to volume comparison is an important factor for volumetric adsorption capacity. The bulk density for the five activated carbons are $A_{1}\left(0.47 \mathrm{~g} / \mathrm{cm}^{3}\right), A_{2}\left(0.39 \mathrm{~g} / \mathrm{cm}^{3}\right), A_{3}\left(0.49 \mathrm{~g} / \mathrm{cm}^{3}\right), A C_{4}\left(0.54 \mathrm{~g} / \mathrm{cm}^{3}\right), A_{5}(0.50$ $\left.\mathrm{g} / \mathrm{cm}^{3}\right)$. Although $\mathrm{AC}_{2}$ has the highest BET surface area and high micropore volume, it has the lowest bulk density. Therefore, it can be concluded that the synergetic effect among BET surface area, micropore volume, micropore size distribution and bulk density play an important role in determining the volumetric adsorption capacity. 


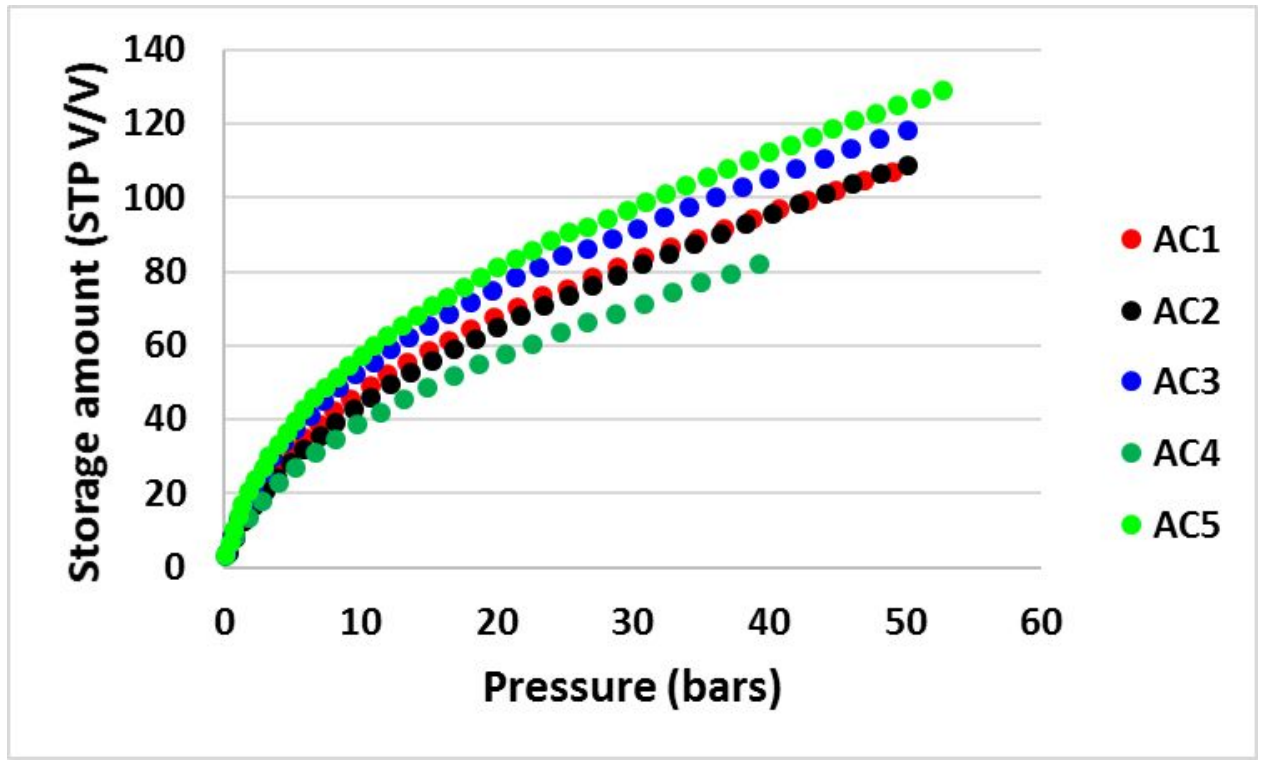

Figure $\mathrm{S}_{3}$. Comparison of volume to volume (v/v) storage capacity among the five granular activated carbons (Data in Table $\mathrm{S}_{3}$ ). 
Table S2. Nitrogen adsorption data at $77 \mathrm{~K}$ for different activated carbons.

\begin{tabular}{|c|c|c|c|c|c|c|c|c|c|}
\hline \multicolumn{2}{|c|}{$\mathrm{AC1}$} & \multicolumn{2}{|c|}{$A C 2$} & \multicolumn{2}{|c|}{ AC3 } & \multicolumn{2}{|l|}{$\mathrm{AC4}$} & \multicolumn{2}{|c|}{ AC5 } \\
\hline $\begin{array}{l}\text { pressure } \\
\text { (P/PO) }\end{array}$ & \begin{tabular}{|l|}
$\begin{array}{l}\text { adsorbed } \\
\text { (mmole/g) }\end{array}$ \\
\end{tabular} & $\begin{array}{l}\text { pressure } \\
(\mathrm{P} / \mathrm{PO})\end{array}$ & \begin{tabular}{|l|} 
adsorbed \\
(mmole/g)
\end{tabular} & $\begin{array}{l}\text { pressure } \\
(\mathrm{P} / \mathrm{P} 0)\end{array}$ & \begin{tabular}{|l|} 
adsorbed \\
$(\mathrm{mmole} / \mathrm{g})$
\end{tabular} & pressure (P/P0) & \begin{tabular}{|l|}
$\begin{array}{l}\text { adsorbed } \\
(\mathrm{mmole} / \mathrm{g})\end{array}$ \\
\end{tabular} & $\begin{array}{l}\text { pressure } \\
(\mathrm{P} / \mathrm{PO})\end{array}$ & \begin{tabular}{|l} 
adsorbed \\
(mmole/g)
\end{tabular} \\
\hline 0.0095344 & 12.01 & 0.0095727 & 13.729 & 0.0094842 & 13.13 & 0.0097981 & 9.1837 & 0.0095079 & 13.55 \\
\hline 0.029519 & 13.331 & 0.028714 & 15.503 & 0.028604 & 14.656 & 0.029547 & 10.118 & 0.02969 & 15.288 \\
\hline 0.060606 & 14.268 & 0.058257 & 17.015 & 0.059989 & 15.81 & 0.059911 & 10.809 & 0.059125 & 16.585 \\
\hline 0.078473 & 14.587 & 0.076373 & 17.659 & 0.077193 & 16.173 & 0.076021 & 11.048 & 0.08061 & 17.173 \\
\hline 0.099546 & 14.85 & 0.097057 & 18.235 & 0.099733 & 16.496 & 0.09907 & 11.308 & 09864 & 17.53 \\
\hline 0.12222 & 15.048 & 0.11882 & 18.708 & 0.12293 & 16.717 & 0.12081 & 11.497 & 12124 & 17.858 \\
\hline 0.14525 & .191 & 14126 & 19.091 & 0.14633 & 16.87 & 0.14276 & 11.649 & 14463 & 18.101 \\
\hline 0.16701 & .29 & 6453 & 19.401 & 0.16856 & 16.975 & 0.16461 & 11.774 & 6817 & 8.277 \\
\hline 0.18533 & 15.355 & 0.18775 & 19.647 & 0.18623 & 17.04 & 0.18426 & 11.87 & 0.18915 & 18.395 \\
\hline 0.20314 & 15.406 & 0.20923 & 19.829 & 0.20339 & 17.092 & 0.20318 & 11.951 & 0.20694 & 18.474 \\
\hline 0.24699 & 15.5 & 0.24904 & 20.081 & 0.24607 & 17.193 & 0.24539 & 12.102 & 0.24761 & 18.61 \\
\hline 0.3001 & 5.577 & 30132 & 20.302 & 0.29935 & 17.285 & 0.2986 & 12.257 & 968 & 18.73 \\
\hline 0.35175 & 15.63 & 0.35618 & 20.452 & 0.34655 & 17.348 & 0.35078 & 12.386 & 0.34575 & 18.81 \\
\hline 0.39739 & 15.667 & 0.39519 & 20.529 & 0.3969 & 17.401 & 0.39642 & 12.486 & 0.39633 & 18.879 \\
\hline 0.44804 & 698 & 0.44635 & 20.607 & 0.4474 & 17.444 & 0.44651 & 12.585 & 0.4469 & 18.936 \\
\hline 0.49784 & 15.722 & 0.49684 & 20.669 & 0.49753 & 17.48 & 0.49647 & 12.678 & 0.49723 & 18.985 \\
\hline 0.54807 & 15.742 & 0.54738 & 20.721 & 0.54783 & 17.51 & 0.54646 & 12.768 & 0.54729 & 19.027 \\
\hline 0.59833 & 15.757 & 0.59745 & 20.766 & 0.59814 & 17.535 & 0.5964 & 12.858 & 0.59751 & 19.064 \\
\hline 0.64826 & 15.77 & 0.6477 & 20.806 & 0.64764 & 17.558 & 0.64631 & 12.951 & 0.6475 & 19.1 \\
\hline 0.69841 & 15.781 & 0.69778 & 20.843 & 0.69803 & 17.581 & 0.69606 & 13.05 & 0.69757 & 19.135 \\
\hline 0.74838 & 15.792 & 0.7477 & 20.882 & 0.7481 & 17.604 & 0.74565 & 13.163 & 0.74747 & 19.173 \\
\hline 0.79839 & 15.805 & 0.79748 & 20.924 & 0.79773 & 17.632 & 0.7948 & 13.299 & 0.79723 & 19.217 \\
\hline 0.81923 & 15.812 & 0.819 & 20.946 & 0.81917 & 17.646 & 0.81772 & 13.377 & 0.81893 & 19.24 \\
\hline 0.84884 & 15.823 & 0.84848 & 20.981 & 0.84857 & 17.671 & 0.84646 & 13.491 & 0.84821 & 19.277 \\
\hline 0.8741 & 15.836 & 0.87332 & 21.019 & 0.87304 & 17.698 & 0.87104 & 13.609 & 0.87338 & 19.314 \\
\hline 0.899 & 15.851 & 0.89824 & 21.069 & 0.89851 & 17.735 & 0.89554 & 13.752 & 0.89806 & 19.36 \\
\hline 0.9236 & 15.874 & 0.92289 & 21.132 & 0.92281 & 17.782 & 0.91994 & 13.925 & 0.92289 & 19.417 \\
\hline 0.94876 & 15.906 & 0.94763 & 21.213 & 0.94786 & 17.844 & 0.94408 & 14.137 & 0.948 & 19.482 \\
\hline 0.97265 & 15.953 & 0.97255 & 21.311 & 0.97327 & 17.91 & 0.97293 & 14.485 & 0.9725 & 19.556 \\
\hline 0.97969 & 15.973 & 0.97952 & 21.345 & 0.97962 & 17.926 & 0.97966 & 14.591 & 0.97937 & 19.584 \\
\hline 0.98894 & 16.01 & 0.98879 & 21.398 & 0.98895 & 17.95 & 0.98765 & 14.748 & 0.9884 & 19.638 \\
\hline 0.9938 & 16.042 & 0.99416 & 21.44 & 0.99432 & 17.965 & 0.99495 & 14.881 & 0.99366 & 19.693 \\
\hline
\end{tabular}


Table S3. Experimental data for activated carbons methane adsorption tests at $21{ }^{\circ} \mathrm{C}$. AC\#-p is for pressure (bars), AC\#-m2m is adsorbed amount (mmole/gram), AC\#-v2v is adsorbed amount based on volume to volume, AC\#-Sv2v is stored amount based on volume to volume.

\begin{tabular}{|c|c|c|c|c|c|c|c|c|c|c|c|c|c|c|c|c|c|c|c|}
\hline AC1-p & AC1-m2m & $A C 1-v 2 v$ & AC1-sv2v & AC2-p & AC2-m2m & $A C 2-v 2 v$ & AC2-sv2v & AC3-p & AC3-m2m & AC3-v2v & AC3-sv2v & AC4-p & AC4-m2m & AC4-v2v & AC4-sv2v & AC5-p & AC5-m2m & AC5-v2v & AC5-sv2v \\
\hline 0.20825 & \begin{tabular}{|l|l|} 
& 0.31741 \\
\end{tabular} & 3.6435 & 3.809 & 0.28425 & \begin{tabular}{|l|}
0.38257 \\
\end{tabular} & 3.644 & 3.8811 & 0.16425 & \begin{tabular}{l|l|}
5 & 0.33323 \\
\end{tabular} & 3.9878 & 4.1184 & 0.12425 & $\begin{array}{ll}0.21965 \\
\end{array}$ & 2.8969 & 2.9884 & 0.09625 & $\begin{array}{ll}0.27158 \\
\end{array}$ & 3.3164 & 3.3914 \\
\hline 0.62525 & 0.65748 & 7.5471 & 8.0444 & 0.82925 & \begin{tabular}{|l|l|} 
& 0.78677 \\
\end{tabular} & 7.494 & 8.1865 & 0.59625 & 0.6719 & 8.0408 & 8.5153 & 0.88925 & 0.57945 & 7.642 & 8.2982 & 0.40825 & 0.53451 & 6.5272 & 6.8456 \\
\hline 1.1694 & 0.97751 & 11.221 & 12.152 & 1.4698 & 1.1692 & 11.137 & 12.366 & 1.0213 & 1.0129 & 12.122 & 12.935 & 1.8023 & 0.90743 & 11.968 & 13.3 & 0.72125 & 0.79799 & \begin{tabular}{l|l|}
9.7448 \\
\end{tabular} & 10.308 \\
\hline 1.6981 & 1.297 & 14.889 & 16.242 & 2.1427 & 1.5465 & 14.73 & 16.524 & 1.562 & 1.3326 & 15.948 & 17.193 & 2.8356 & 1.214 & 16.01 & 18.11 & 1.0373 & $\begin{array}{l}1.0597 \\
\end{array}$ & 12.94 & 13.75 \\
\hline 2.363 & 1.5965 & 18.326 & 20.211 & 3.0078 & 1.8855 & 17.96 & 20.481 & 2.1988 & 1.6384 & 19.607 & 21.362 & 3.9971 & 1.4935 & 19.697 & 22.663 & 1.3697 & 1.3189 & 16.105 & 17.175 \\
\hline 3.136 & 1.8785 & 21.563 & 24.068 & 3.8369 & 2.2297 & 21.238 & 24.46 & 2.8796 & 1.9368 & 23.179 & 25.479 & 5.2867 & 1.7428 & 22.985 & 26.917 & 1.7983 & 1.5662 & 19.126 & 20.532 \\
\hline 4.0251 & 2.142 & 24.588 & 27.809 & 4.8782 & 2.5281 & 24.08 & 28.183 & 3.5565 & 2.2357 & 26.755 & 29.6 & 6.6885 & 1.97 & 25.982 & 30.969 & 2.3389 & 1.7941 & 21.908 & 23.739 \\
\hline 4.9663 & 2.3957 & 27.5 & 31.481 & 5.8955 & 2.8327 & 26.981 & 31.949 & 4.4336 & 2.5038 & 29.964 & 33.516 & 8.1784 & 2.1783 & 28.729 & 34.843 & 2.8636 & 2.0253 & 24.732 & 26.976 \\
\hline 5.8875 & 2.6535 & 30.459 & 35.186 & 7.0209 & 3.114 & 29.661 & 35.59 & 5.2707 & 2.7767 & 33.229 & 37.459 & 9.7645 & \begin{tabular}{l|}
2.3658 \\
\end{tabular} & 31.202 & 38.523 & 3.3162 & 2.2673 & $\begin{array}{l}27.687 \\
\end{array}$ & 30.287 \\
\hline 6.9569 & 2.8889 & 33.162 & 38.758 & 8.2145 & 3.3826 & 32.219 & 39.17 & 6.3361 & 3.0124 & 36.05 & 41.144 & 11.431 & 2.5375 & 33.466 & 42.062 & 3.969 & 2.4779 & 30.259 & 33.374 \\
\hline 8.0983 & 3.1111 & 35.711 & 42.239 & 9.4801 & 3.6335 & 34.609 & 42.649 & 7.3814 & 3.251 & 38.906 & 444.852 & 13.173 & 2.6905 & 35.484 & 45.42 & \begin{tabular}{|l|l|}
4.6259 \\
\end{tabular} & 2.6891 & \begin{tabular}{l|l}
32.839 \\
\end{tabular} & 36.473 \\
\hline 9.3199 & 3.3201 & 38.112 & 45.641 & 10.814 & 3.8675 & 36.839 & 46.032 & 8.4868 & 3.4782 & 41.625 & 48.475 & 14.971 & 2.8306 & 37.331 & \begin{tabular}{|l|}
48.661 \\
\end{tabular} & 5.2787 & 2.8996 & 35.409 & 39.561 \\
\hline 10.706 & 3.5007 & 40.184 & 48.854 & 12.208 & 4.0917 & 38.974 & 49.378 & 9.6843 & 3.6929 & 44.194 & 52.027 & 16.829 & 2.9551 & 38.973 & 51.751 & 5.8915 & 3.1159 & 38.051 & 42.69 \\
\hline 12.023 & 3.6948 & 42.413 & 52.173 & 13.71 & 4.2922 & 40.883 & 52.599 & 10.942 & 3.8959 & 46.624 & 55.494 & 18.732 & 3.0707 & 40.498 & 54.768 & 6.6364 & 3.3147 & 40.478 & 45.711 \\
\hline 13.469 & 3.8664 & 44.382 & 55.344 & 15.272 & 4.4756 & 42.63 & 55.716 & 12.26 & 4.0887 & 48.931 & 58.893 & 20.678 & 3.1774 & 41.905 & 57.712 & 7.4575 & 3.4989 & \begin{tabular}{|l|}
42.727 \\
\end{tabular} & 48.616 \\
\hline 15.019 & 4.0203 & \begin{tabular}{|l|l|}
46.149 \\
\end{tabular} & 58.406 & 16.834 & \begin{tabular}{|l|}
4.6597 \\
\end{tabular} & 44.383 & 58.848 & 13.605 & 4.275 & 51.16 & 62.242 & 22.657 & 3.2739 & 43.178 & 60.558 & 8.3186 & 3.6783 & \begin{tabular}{|l|}
44.918 \\
\end{tabular} & 51.497 \\
\hline 16.533 & 4.178 & \begin{tabular}{ll|}
47.959 \\
\end{tabular} & 61.488 & 18.444 & \begin{tabular}{|l|l|}
4.8327 \\
\end{tabular} & 46.032 & 61.926 & 15.051 & \begin{tabular}{|l|l|}
4.4471 \\
\end{tabular} & 53.22 & 65.511 & 24.672 & $\begin{array}{l}3.3597 \\
\end{array}$ & $\begin{array}{l}44.309 \\
\end{array}$ & 63.302 & \begin{tabular}{|c|}
9.1837 \\
\end{tabular} & 3.8569 & $\begin{array}{l}47.099 \\
\end{array}$ & 54.374 \\
\hline 18.155 & 4.3204 & 49.593 & 64.492 & 20.098 & 4.9953 & 47.58 & 64.95 & 16.565 & 4.606 & 55.122 & 68.685 & 26.71 & 3.4422 & 45.397 & 66.032 & 10.093 & 4.0272 & 49.178 & 57.186 \\
\hline 19.837 & $\begin{array}{l}4.4507 \\
\end{array}$ & \begin{tabular}{|l|l|}
51.089 \\
\end{tabular} & 67.417 & 21.796 & 5.149 & 49.045 & 67.939 & 18.127 & $\begin{array}{l}4.7563 \\
\end{array}$ & 56.921 & 71.805 & 28.761 & \begin{tabular}{|l|}
3.5188 \\
\end{tabular} & \begin{tabular}{ll|}
46.408 \\
\end{tabular} & \begin{tabular}{|l|l|}
68.707 \\
\end{tabular} & 11.05 & \begin{tabular}{|l|}
4.1913 \\
\end{tabular} & 51.182 & 59.965 \\
\hline 21.564 & \begin{tabular}{|l|}
4.573 \\
\end{tabular} & 52.493 & 70.296 & 23.53 & 5.2904 & 50.392 & 70.851 & 19.749 & 4.896 & 58.592 & 74.854 & 30.835 & 3.5887 & 47.33 & 71.323 & 12.035 & 4.3495 & 53.114 & 62.697 \\
\hline 23.338 & 4.7243 & 54.229 & 73.558 & 25.296 & 5.376 & 51.207 & 73.27 & 21.415 & 5.0598 & 60.552 & 78.238 & 32.926 & 3.6921 & 48.693 & 74.406 & 13.065 & 4.5006 & 54.96 & 65.381 \\
\hline 25.152 & 4.7519 & 54.546 & 75.444 & 27.095 & 5.5068 & 52.452 & 76.159 & 23.138 & 5.1844 & 62.043 & 81.209 & 35.033 & $\begin{array}{l}3.7557 \\
\end{array}$ & 49.532 & \begin{tabular}{|l|}
76.988 \\
\end{tabular} & $\begin{array}{l}14.142 \\
\end{array}$ & $\begin{array}{l}4.6466 \\
\end{array}$ & \begin{tabular}{|l|}
56.743 \\
\end{tabular} & 68.045 \\
\hline 27.003 & 4.8541 & 55.719 & 78.227 & 28.917 & 5.6311 & 53.636 & 79.017 & 24.884 & 5.304 & 63.475 & 84.151 & 37.156 & 3.8141 & 50.303 & 79.528 & 15.247 & 4.7855 & 58.438 & 70.647 \\
\hline 28.893 & 4.9486 & 56.805 & 80.967 & 30.771 & 5.7471 & 54.741 & 81.836 & 26.674 & 5.3333 & 63.825 & 86.058 & 39.266 & 3.8735 & 51.086 & 82.082 & \begin{tabular}{|c|}
16.437 \\
\end{tabular} & 4.9121 & 59.985 & 73.175 \\
\hline 30.811 & 5.0375 & 57.825 & 83.677 & 32.642 & 5.8582 & 55.8 & 84.635 & 28.504 & 5.437 & 65.066 & 88.901 & & & & & 17.566 & 5.0463 & 61.624 & 75.748 \\
\hline 32.762 & 5.1199 & 58.771 & 86.353 & 34.532 & 5.9636 & 56.803 & 87.407 & 30.371 & 5.5336 & 66.223 & 91.7 & & & & & 18.78 & 5.1923 & 63.406 & 78.538 \\
\hline 34.748 & 5.1953 & 59.636 & 88.989 & 36.443 & 6.0632 & 57.752 & 90.154 & 32.261 & 5.6252 & 67.319 & \begin{tabular}{|l|}
94.47 \\
\end{tabular} & & & & & 20.082 & 5.3028 & \begin{tabular}{l|l|}
64.756 \\
\end{tabular} & 80.974 \\
\hline 36.751 & 5.2668 & \begin{tabular}{|c|}
60.457 \\
\end{tabular} & 91.608 & 38.373 & $\begin{array}{l}6.157 \\
\end{array}$ & 58.645 & 92.876 & 34.18 & 5.7109 & 68.345 & 97.206 & & & & & 21.351 & 5.4179 & 66.161 & 83.443 \\
\hline 38.762 & 5.3358 & 61.249 & 94.217 & 40.312 & 6.2476 & 59.508 & 95.586 & 36.122 & 5.7915 & 69.309 & 99.912 & & & & & 22.661 & 5.5262 & 67.484 & 85.868 \\
\hline 40.8 & 5.399 & \begin{tabular}{|c|}
61.974 \\
\end{tabular} & 96.794 & 42.266 & 6.3332 & 60.324 & 98.274 & 38.073 & 5.8697 & 70.245 & 102.61 & & & & & 23.991 & 5.6309 & \begin{tabular}{l|l}
68.763 \\
\end{tabular} & $\begin{array}{l}88.27 \\
\end{array}$ \\
\hline 42.859 & 5.4576 & 62.647 & 99.35 & 44.225 & 6.4165 & 61.118 & 100.96 & 40.043 & 5.9433 & 71.126 & 105.28 & & & & & 25.34 & 5.732 & 69.997 & 90.651 \\
\hline 44.905 & 5.5171 & 63.33 & 101.92 & 46.203 & 6.494 & 61.855 & 103.61 & 42.034 & 6.0124 & 71.952 & 107.92 & & & & & 26.734 & 5.7474 & 70.185 & 92.027 \\
\hline 46.98 & 5.5707 & 63.945 & 104.45 & 48.182 & 6.57 & 62.58 & 106.26 & 44.048 & 6.0762 & 72.716 & 110.53 & & & & & 28.136 & 5.8392 & 71.306 & 94.35 \\
\hline \multirow[t]{16}{*}{49.083} & 5.6183 & 64.492 & 106.96 & 50.172 & 6.642 & 63.265 & 108.9 & 46.075 & 6.1369 & 73.442 & 113.13 & & & & & 29.562 & 5.9267 & 72.375 & 96.646 \\
\hline & & & & & & & & 48.11 & 6.195 & 74.138 & 115.72 & & & & & 31.012 & 6.0098 & $\begin{array}{l}73.39 \\
\end{array}$ & 98.915 \\
\hline & & & & & & & & 50.14 & 6.2527 & 74.828 & 118.31 & & & & & 32.482 & 6.0892 & 74.359 & 101.16 \\
\hline & & & & & & & & & & & & & & & & 33.963 & 6.1661 & $\begin{array}{l}75.297 \\
\end{array}$ & 103.39 \\
\hline & & & & & & & & & & & & & & & & 35.465 & 6.2391 & $\begin{array}{l}76.19 \\
\end{array}$ & 105.6 \\
\hline & & & & & & & & & & & & & & & & 36.979 & $\begin{array}{l}6.3097 \\
\end{array}$ & 77.051 & 107.8 \\
\hline & & & & & & & & & & & & & & & & 38.509 & \begin{tabular}{|l|}
6.377 \\
\end{tabular} & \begin{tabular}{|l|}
77.873 \\
\end{tabular} & 109.98 \\
\hline & & & & & & & & & & & & & & & & 40.043 & 6.4431 & \begin{tabular}{|l|}
78.68 \\
\end{tabular} & 112.15 \\
\hline & & & & & & & & & & & & & & & & 41.601 & $\begin{array}{l}6.5047 \\
\end{array}$ & 79.432 & 114.29 \\
\hline & & & & & & & & & & & & & & & & 43.163 & $\begin{array}{ll}6.565 \\
\end{array}$ & 80.169 & 116.43 \\
\hline & & & & & & & & & & & & & & & & 44.733 & 6.6234 & 80.882 & 118.56 \\
\hline & & & & & & & & & & & & & & & & 46.315 & 6.6791 & $\begin{array}{l}81.563 \\
\end{array}$ & 120.67 \\
\hline & & & & & & & & & & & & & & & & 47.913 & 6.7317 & 82.205 & 122.77 \\
\hline & & & & & & & & & & & & & & & & 49.507 & 6.7843 & 82.847 & 124.87 \\
\hline & & & & & & & & & & & & & & & & 51.113 & 6.8342 & \begin{tabular}{|l|}
83.457 \\
\end{tabular} & 126.95 \\
\hline & & & & & & & & & & & & & & & & 52.727 & 6.8823 & 84.044 & 129.03 \\
\hline
\end{tabular}

Table S4. Experimental and model data for Figure 3 (AC5-methane system). 


\begin{tabular}{|c|c|c|c|c|c|c|c|}
\hline \multicolumn{2}{|r|}{$10^{\circ} \mathrm{C}$} & \multicolumn{2}{|r|}{$21^{\circ} \mathrm{C}$} & \multicolumn{2}{|c|}{$38^{\circ} \mathrm{C}$} & \multicolumn{2}{|c|}{$56^{\circ} \mathrm{C}$} \\
\hline Presure (bar) & $\begin{array}{l}\text { Adsorbed amount } \\
\text { (mmole/g) }\end{array}$ & Presure (bar) & $\begin{array}{l}\text { Adsorbed amount } \\
\text { (mmole/g) }\end{array}$ & Presure $(\mathrm{b}$ & $\begin{array}{l}\text { Adsorbed } \\
\text { amount } \\
\text { (mmole/ } \\
\text { g) }\end{array}$ & Presure (bar) & $\begin{array}{l}\text { Adsorbe } \\
\text { d } \\
\text { amount } \\
\text { (mmole/ } \\
\text { g) }\end{array}$ \\
\hline 0.06025 & 0.30914 & 0.09625 & 0.27157 & 0.16425 & 0.19688 & 0.30825 & 0.20968 \\
\hline 0.26425 & 0.58733 & 0.40825 & 0.53448 & 0.48025 & 0.46026 & 0.73725 & 0.45941 \\
\hline 0.56825 & 0.85126 & 0.72125 & 0.79794 & 0.90125 & 0.70965 & 1.2816 & 0.6913 \\
\hline 0.88925 & 1.1115 & 1.0373 & 1.0596 & 1.3577 & 0.9535 & 1.9224 & 0.91072 \\
\hline 1.2095 & 1.3717 & 1.3697 & 1.3188 & 1.9064 & 1.1839 & 2.4711 & 1.142 \\
\hline 1.5379 & 1.6319 & 1.7983 & 1.5661 & 2.359 & 1.427 & 3.156 & 1.3552 \\
\hline 1.9585 & 1.8778 & 2.3389 & 1.7939 & 2.9998 & 1.6437 & 3.8889 & 1.5614 \\
\hline 2.387 & 2.1211 & 2.8636 & 2.0251 & 3.6366 & 1.8611 & 4.726 & 1.7512 \\
\hline 2.8316 & 2.3646 & 3.3162 & 2.2671 & 4.3135 & 2.0711 & 5.5551 & 1.9433 \\
\hline 3.2882 & 2.6035 & 3.969 & 2.4776 & 5.0384 & 2.2753 & 6.4162 & 2.1296 \\
\hline 3.8489 & 2.8263 & 4.6259 & 2.6888 & 5.8675 & 2.4626 & 7.3293 & 2.3095 \\
\hline 4.4216 & 3.0496 & 5.2787 & 2.8992 & 6.6725 & 2.6534 & 8.2866 & 2.483 \\
\hline 5.0584 & 3.2591 & 5.8915 & 3.1155 & 7.5296 & 2.839 & 9.2718 & 2.6522 \\
\hline 5.6632 & 3.4748 & 6.6364 & 3.3142 & 8.4227 & 3.0163 & 10.301 & 2.8124 \\
\hline 6.3561 & 3.6792 & 7.4575 & 3.4983 & 9.3599 & 3.1885 & 11.383 & 2.9688 \\
\hline 7.089 & 3.8758 & 8.3186 & 3.6776 & 10.333 & 3.355 & 12.448 & 3.1248 \\
\hline 7.9021 & 4.0598 & 9.1837 & 3.8561 & 11.342 & 3.5146 & 13.641 & 3.2634 \\
\hline 8.7191 & 4.2417 & 10.093 & 4.0263 & 12.4 & 3.6683 & 14.831 & 3.4024 \\
\hline 9.5842 & 4.4158 & 11.05 & 4.1904 & 13.497 & 3.8144 & 16.097 & 3.5289 \\
\hline 10.493 & 4.5854 & 12.035 & 4.3485 & 14.575 & 3.9633 & 17.346 & 3.6588 \\
\hline 11.439 & 4.7464 & 13.065 & 4.4995 & 15.792 & 4.0936 & 18.636 & 3.7813 \\
\hline 12.424 & 4.8995 & 14.142 & 4.6454 & 16.986 & 4.2247 & 19.949 & 3.9001 \\
\hline 13.449 & 5.0485 & 15.247 & 4.7841 & 18.175 & 4.3573 & 21.311 & 4.0143 \\
\hline 14.515 & 5.1909 & 16.437 & 4.9107 & 19.489 & 4.4711 & 22.689 & 4.1233 \\
\hline 15.72 & 5.3094 & 17.566 & 5.0448 & 20.783 & 4.5861 & 24.095 & 4.2293 \\
\hline 16.805 & 5.4394 & 18.78 & 5.1906 & 22.116 & 4.7011 & 25.529 & 4.3041 \\
\hline 17.983 & 5.5651 & 20.082 & 5.301 & 23.474 & 4.8094 & 26.986 & 4.4042 \\
\hline 19.249 & 5.6762 & 21.351 & 5.416 & 24.852 & 4.9143 & 28.46 & 4.5017 \\
\hline 20.482 & 5.7919 & 22.661 & 5.5242 & 26.266 & 5.0134 & 29.966 & 4.5942 \\
\hline 21.764 & 5.8993 & 23.991 & 5.6287 & 27.695 & 5.1096 & 31.488 & 4.6841 \\
\hline 23.057 & 6.0042 & 25.34 & 5.7297 & 29.157 & 5.2006 & 33.03 & 4.7707 \\
\hline 24.387 & 6.1027 & 26.734 & 5.7449 & 30.631 & 5.2894 & 34.596 & 4.8535 \\
\hline 25.741 & 6.1967 & 28.136 & 5.8365 & 32.121 & 5.3753 & 36.17 & 4.9348 \\
\hline 27.115 & 6.2868 & 29.562 & 5.9239 & 33.647 & 5.4553 & 37.776 & 5.011 \\
\hline 28.516 & 6.3717 & 31.012 & 6.0068 & 35.181 & 5.5336 & 39.394 & 5.0852 \\
\hline 29.942 & 6.4519 & 32.482 & 6.086 & 36.735 & 5.6084 & 41.012 & 5.1591 \\
\hline 31.368 & 6.5314 & 33.963 & 6.1626 & 38.297 & 5.6815 & 42.655 & 5.2291 \\
\hline 32.83 & 6.6043 & 35.465 & 6.2355 & 39.875 & 5.7517 & 44.301 & 5.2982 \\
\hline 34.3 & 6.6752 & 36.979 & 6.3058 & 41.477 & 5.7395 & 45.963 & 5.3646 \\
\hline 35.794 & 6.7412 & 38.509 & 6.373 & 43.079 & 5.8051 & 47.641 & 5.4283 \\
\hline 37.304 & 6.8039 & 40.043 & 6.4389 & 44.697 & 5.8678 & 49.319 & 5.4917 \\
\hline 38.814 & 6.8658 & 41.601 & 6.5002 & 46.311 & 5.9307 & 51.009 & 5.5531 \\
\hline 40.352 & 6.8439 & 43.163 & 6.5603 & 47.941 & 5.9906 & 52.703 & 5.6135 \\
\hline 41.886 & 6.9001 & 44.733 & 6.6185 & 49.575 & 6.0495 & & \\
\hline 43.428 & 6.9542 & 46.315 & 6.674 & 51.218 & 6.1067 & & \\
\hline 44.994 & 7.0034 & 47.913 & 6.7263 & 52.876 & 6.1609 & & \\
\hline 46.552 & 7.0532 & 49.507 & 6.7786 & 54.542 & 6.2135 & & \\
\hline 48.13 & 7.0988 & 51.113 & 6.8284 & & & & \\
\hline 49.708 & 7.1435 & 52.727 & 6.8761 & & & & \\
\hline 51.282 & 7.1881 & & & & & & \\
\hline
\end{tabular}



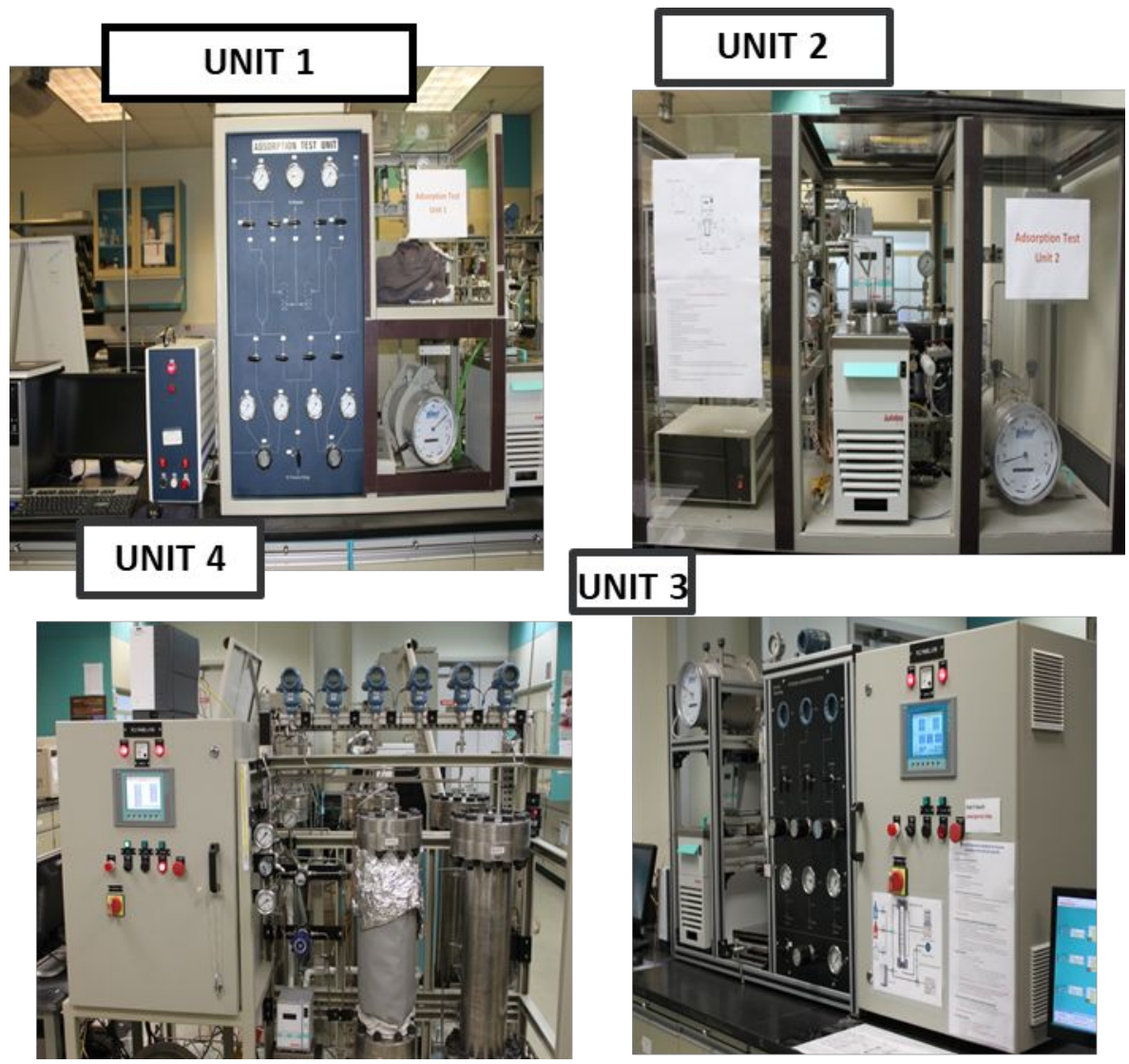

Figure s4. Pictorial view of gas adsorption lab setup.

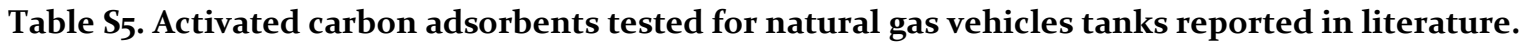

\begin{tabular}{|l|l|l|l|}
\hline Project & Investigation Method & Pressure (bar) & $\begin{array}{l}\text { Tank Uptake (volume to } \\
\text { volume) }\end{array}$ \\
\hline $\begin{array}{l}\text { Atlanta Gas Light } \\
\text { Adsorbent Research Group }\end{array}$ & $\begin{array}{l}\text { Chrysler B Van, Dodge } \\
\text { Dakota truck }\end{array}$ & $35-40$ & $\begin{array}{l}150 \text { in laboratory } \\
\text { conditions }\end{array}$ \\
\hline EU FPS LEVINGS program & $\begin{array}{l}\text { FIAT Marea on board, } \\
\text { field testing }\end{array}$ & $35-40$ & 123 \\
\hline $\begin{array}{l}\text { Oak Ridge National } \\
\text { Laboratory }\end{array}$ & Lab Investigation & 35 & 150 \\
\hline $\begin{array}{l}\text { Honda Motors } \\
\text { University of Petroleum, } \\
\text { China }\end{array}$ & $\begin{array}{l}\text { Car Xiali 7131U on } \\
\text { board field testing }\end{array}$ & 50 & 155 \\
\hline $\begin{array}{l}\text { University of Petroleum, } \\
\text { China }\end{array}$ & $\begin{array}{l}\text { Car Xiali 7131U on } \\
\text { board field testing }\end{array}$ & 125 & $170-180$ \\
\hline $\begin{array}{l}\text { Brazilian Gas Technology } \\
\text { Center }\end{array}$ & $\begin{array}{l}\text { Lab investigation on } \\
\text { full size prototype }\end{array}$ & $35-40$ & $130-150$ \\
\hline
\end{tabular}




\begin{tabular}{|l|l|l|l|}
\hline $\begin{array}{l}\text { ALL-CRAFT (University of } \\
\text { Missouri }\end{array}$ & $\begin{array}{l}\text { Lab investigation, } 35 \\
\text { adsorbent } \\
\text { optimization, field } \\
\text { testing }\end{array}$ & $\begin{array}{l}\text { Intragranular capability: } \\
\text { 202; Monolith capability: } \\
161\end{array}$ \\
\hline $\begin{array}{l}\text { ALL-CRAFT (University of } \\
\text { Missouri }\end{array}$ & $\begin{array}{l}\text { Lab investigation, } \\
\text { adsorbent } \\
\text { optimization, field } \\
\text { testing }\end{array}$ & 250 & $\begin{array}{l}\text { Intragranular capability: } \\
\text { 337; Monolith capability: to } \\
\text { be determined }\end{array}$ \\
\hline
\end{tabular}

